\title{
Serum Uric Acid Is Associated with Metabolic Syndrome and Insulin Resistance among Health Personnel from Peru
}

\author{
Brenda M. Galindo-Yllu, ${ }^{1}$ Ricardo Rojas-Humpire ${ }^{(D,},{ }^{1}$ Carlos J. Toro-Huamanchumo, ${ }^{2}$ \\ Rosmery Gutierrez-Ajalcriña, ${ }^{3}$ and Anderson N. Soriano ${ }^{1,4}$ \\ ${ }^{1}$ Clinical and Epidemiological Research Unit, School of Medicine, Universidad Peruana Union, Lima, Peru \\ ${ }^{2}$ Universidad San Ignacio de Loyola, Unidad para la Generación y Síntesis de Evidencias en Salud, Lima, Peru \\ ${ }^{3}$ Unidad de Epidemiología y Salud Ambiental, Hospital de Huaycán, Ate, Peru \\ ${ }^{4}$ Research Coordination, Clínica Good Hope, Lima, Peru
}

Correspondence should be addressed to Anderson N. Soriano; andsor19@gmail.com

Received 15 March 2021; Revised 8 August 2021; Accepted 4 September 2021; Published 15 November 2021

Academic Editor: Abdah Md Akim

Copyright ( 92021 Brenda M. Galindo-Yllu et al. This is an open access article distributed under the Creative Commons Attribution License, which permits unrestricted use, distribution, and reproduction in any medium, provided the original work is properly cited.

We explored the association between serum uric acid (SUA) and metabolic syndrome (MetS) and insulin resistance (IR) among health personnel from a public hospital in Peru in a cross-sectional study with data from the Plan for the Prevention and Surveillance of Communicable and Noncommunicable Diseases of Huaycán Hospital. MetS was defined according to Latin American Diabetes Association (ALAD) criteria and IR with surrogate IR markers, triglyceride-to-HDL-C ratio (TG/ HDL-C), and triglyceride-to-glucose index (TyG). The association between SUA and MetS and IR was determined using Poisson regression models in a sample of 292 participants with an average age of $46.2 \pm 10.6$ years. The total prevalence of MetS was 38\%, and the individuals with MetS presented mainly alterations in anthropometric parameters (obesity and body fat). Finally, the adjusted regression models showed that women with SUA in the highest tertile increased the prevalence of MetS (PR: 1.71, 95\% CI: 1.07-2.74) compared to the lowest tertile of SUA in women, while SUA increased hypertriglyceridemia and IR (TG/HDL-C and TyG) in both sexes. We concluded that SUA is strongly associated with MetS in women, and SUA increases hypertriglyceridemia and IR in both sexes. On the contrary, more research is required regarding the female population.

\section{Introduction}

In recent decades, metabolic syndrome (MetS) has increased dramatically and is considered one of the most important risk factors for cardiovascular disease [1]. MetS is a set of interrelated clinical disorders, including dyslipidemia, central obesity, glucose intolerance, and high blood pressure [2]. Its presence is involved in the development of various diseases such as fatty liver, diabetes mellitus (DM), cancer, and cardiovascular and infectious diseases $[3,4]$. Prior research indicates that insulin resistance (IR) plays an important role in the pathophysiology of this condition [5]. Considering this, TyG and TG/HDL ratio are simple and practical surrogate markers for insulin resistance that can be used in primary healthcare [6].
Serum uric acid (SUA) is an excretory metabolite produced by the metabolism of the purines [7]. It can be elevated as a result of the low renal filtration rate, overproduction of purine precursors, and diet [8]. SUA regulates proinflammatory pathways in vascular smooth muscle cells and oxidative stress at the mitochondrial level [9]. Additionally, it is involved in the mechanisms of metabolic dysregulation mediated by excess fructose [10]. Previous studies have reported the association of SUA concentrations and MetS, IR, DM, and other cardiometabolic diseases $[11,12]$.

Several Latin American countries have exhibited a high prevalence of MetS [13]. The biological diversity of the Latino population leads to changes in the prevalence and development of certain diseases such as metabolic diseases 
[14]. Several risk factors have been studied; for example, a study in Brazilian women found that anthropometric parameters and estradiol were strongly associated to MetS [15]; however, SUA was not evaluated in that study and could be an important factor to consider. On the contrary, healthcare personnel are important agents of public health and quality of life in communities [16]. Moreover, the work pace of health personnel, bad sleep quality, stress, and anxiety could increase the risk of cardiometabolic disorders such as diabetes, hypertension, IR, and MetS [17]. For this reason, we found it appropriate to conduct a study in a Latin population, specifically in a Peruvian hospital, to analyze the association of MetS with SUA and IR stratified by sex in health personnel.

\section{Methods}

2.1. Design of the Primary Study. We conducted a crosssectional analytical study using data from adult workers of both sexes who were part of the Plan for the Prevention and Surveillance of Communicable and Noncommunicable Diseases at Hospital de Huaycán II-1, Lima, Peru, in 2019. In this prevention plan, clinical evaluation and laboratory tests are performed to prevent and diagnose diseases in workers. Trained personnel collected data on demographic characteristics, lifestyle behaviors, and anthropometric measurements and laboratory data using questionnaires. Likewise, workers were explained that their medical data would be used for future research, and written informed consent was obtained from all participants.

2.2. Eligibility Criteria. We included health personnel data from Hospital de Huaycan. Pregnant women, participants who did not fill out the form, participants who did not perform laboratory tests, and those who did not present plausible data for the study were excluded. We eliminated 75 observations due to missing data $(n=46,12.5 \%)$ and implausible data $(n=29,7.9 \%)$ (Table S1 in Supplementary Materials).

\subsection{Definition of Variables}

2.3.1. Exposure: SUA. SUA concentration was categorized into tertiles according to sex-specific distribution: T1 $(2.5-4.4 \mathrm{mg} / \mathrm{dL}), \mathrm{T} 2(4.5-5.0 \mathrm{mg} / \mathrm{dL})$, and T3 $(5.1-6.3 \mathrm{mg} /$ $\mathrm{dL})$ for men and $\mathrm{T} 1(1.2-3.1 \mathrm{mg} / \mathrm{dL}), \mathrm{T} 2(3.2-3.8 \mathrm{mg} / \mathrm{dL})$, and T3 $(3.9-6.8 \mathrm{mg} / \mathrm{dL})$ for women.

2.3.2. Outcome: MetS. MetS was defined according to the criteria of Latin American Diabetes Association (ALAD) 2010 [18], including waist circumference (WC), triglycerides (TG), high-density lipoprotein cholesterol (HDL-C), systolic blood pressure (SBP), diastolic blood pressure (DBP), fasting glucose (FG), glycosylated hemoglobin (HbA1c), and some data from the workers' registry. The criteria for SM were central obesity ( $\mathrm{WC} \geq 94 \mathrm{~cm}$ in men and $\geq 88 \mathrm{~cm}$ in women) and two or more of the following: hypertriglyceridemia (TG $>150 \mathrm{mg} / \mathrm{dL}$ or in specific hypolipidemic therapy), low
HDL-C (HDL-C $<40 \mathrm{mg} / \mathrm{dL}$ in men and $<50 \mathrm{mg} / \mathrm{dL}$ in women), high blood pressure (SBP $\geq 130 \mathrm{mmHg}$ and/or SBP $\geq 85 \mathrm{mmHg}$ or on antihypertensive treatment), or impaired glucose regulation ( $\mathrm{SBP} \geq 100 \mathrm{mg} / \mathrm{dL}, \mathrm{HbAlc}>5.6 \%$, or in treatment for DM).

2.3.3. Other Variables. Triglyceride-to-HDL cholesterol ratio (TG/HDL-C) and triglyceride-to-glucose index (TyG) were selected as insulin resistance indexes because they are accurate indicators of insulin resistance diagnosis in many populations [19]. TG/HDL-C was calculated using the following formula: fasting TG $(\mathrm{mg} / \mathrm{dL}) /$ fasting HDL cholesterol $(\mathrm{mg} / \mathrm{dL})$, and it was categorized into elevated $(\geq 3)$ and normal TG/HDL-C [20]. TyG was calculated using the formula $\operatorname{Ln}$ [fasting TG $(\mathrm{mg} / \mathrm{dL}) \times$ fasting plasma glucose $(\mathrm{mg} / \mathrm{dL}) / 2]$ and categorized into elevated $(\geq 8.65)$ and normal TyG [21].

Nutrition, smoking, alcohol consumption, and physical activity were extracted from the Fantastico, a healthy lifestyle questionnaire, validated in Peru [22]. The variables were categorized into "good" nutrition (balanced diet almost always and no consumption of sugar, salt, junk food, or high fat) and "bad" nutrition (balanced diet sometimes or rarely and consumption of sugar, salt, junk food, and/or high fat), "physically active" (active exercise at least 20 min four or more times per week) and "physically inactive" (active exercise at least $20 \mathrm{~min}$ one to three times per week or less than one time per week), "low-moderate alcohol consumption" (0-7 drinks per week) and "high alcohol consumption" (8-12 or more drinks per week), and "nonsmoking" (no smoking in the last year) and "smoking" (smoked this year or smokes 1-10 cigarettes per day or more than 10 per day). The family history of DM was extracted from the FINDRISC questionnaire and was categorized as "Yes" and "No." The variables of age, sex, type of employee, BMI, low-density lipoprotein cholesterol (LDL-C), very low-density lipoprotein cholesterol (VLDL-C), total cholesterol, and body fat percentage were also included.

2.4. Statistical Analysis. Data analysis was performed with RStudio v1.3 software. Categorical variables were described in absolute and relative frequencies. Numerical variables were described with mean and standard deviation. To assess the association between the SUA level (as a numerical value and categorized by tertiles) and metabolic syndrome, prevalence ratios (PRs) and their respective 95\% confidence intervals (95\% CIs) were determined using Poisson regression models with robust variance. The first model examined the bivariate association between SUA and metabolic syndrome. The second model was adjusted for age and sex in the overall population and by age in the sex-stratified population. The third model was additionally adjusted for BMI variables, nutrition, smoking, alcohol consumption, physical activity, and family history of DM. In the same way, the analysis of MetS components and insulin resistance markers had adjustments for these potential confounders. The association analysis was stratified by sex, and a $p$ value $<0.05$ was considered statistically significant. 
2.5. Ethical Considerations. The study was approved by the Institutional Review Board of Hospital de Huaycán (no. 0232020) and of the Faculty of Health Sciences of the Universidad Peruana Unión (no. 00136-2020).

\section{Results}

3.1. General Characteristics of the Study Population. We analyzed the data from a total of 292 individuals (202 women and $90 \mathrm{men})$. The mean age of the participants was $46.2 \pm 10.6$ years, and workers were in administrative (25.3\%), patient contact $(66.5 \%)$, and general services $(8.2 \%)$ positions. The largest proportion was nonsmokers (87.7\%), had good nutrition $(71.6 \%)$, had no family history of DM (65.1\%), reported low-moderate alcohol consumption (93.5\%), and were physically active (81.5\%). The mean concentration of SUA was $3.9 \pm 1.0 \mathrm{mg} / \mathrm{dL}$ in the general population, $\quad 3.49 \pm 0.88 \mathrm{mg} / \mathrm{dL}$ in women, and $4.70 \pm 0.85 \mathrm{mg} / \mathrm{dL}$ in men. The characteristics of both MetS and no-MetS groups are presented in Table S2 in Supplementary Materials.

The patient contact personnel proportion was the highest in the group with MetS, with ages between 50 and 60 years. The MetS group showed important anthropometric alterations, obesity, and visceral fat. Furthermore, glucose metabolism (HbAlc: 6.6\% in men and 6.2\% in women; both $p<0.05)$, lipid profile, triglycerides $(234 \mathrm{mg} / \mathrm{dL}$ in men and $188 \mathrm{mg} / \mathrm{dL}$ in women; both $p<0.05)$, total cholesterol $(216 \mathrm{mg} / \mathrm{dL}$ in men and $199 \mathrm{mg} / \mathrm{dL}$ in women; both $p<0.05)$, VLDL-C $(40.5 \mathrm{mg} / \mathrm{dL}$ in men and $36.6 \mathrm{mg} / \mathrm{dL}$ in women; both $p<0.05$ ), and blood pressure presented significant differences in the MetS group compared to the noMetS group in both sexes. However, some laboratory tests in the MetS group presented significant changes only in women, such as HDL-C (53 vs. $47.8 \mathrm{mg} / \mathrm{dL}, p<0.01)$ and uric acid ( 3.3 vs. $3.8 \mathrm{mg} / \mathrm{dL}, p<0.01)$, while only in men, changes in LDL-C (113 vs. $129 \mathrm{mg} / \mathrm{dL}, p<0.05)$ were shown (Table S2 in Supplementary Materials).

3.2. MetS Prevalence by Tertiles of SUA Concentration. The prevalence of MetS in the total population was $38 \%$, $36.7 \%$ in men, and $38.6 \%$ in women. The components of MetS changed by each SUA tertile. In this way, more MetS components were pooled in the high tertile than in the low tertile in both men ( 24.1 vs. $12.1 \%)$ and women (31.3 vs. $6.8 \%)$, as shown in Figure 1.

\subsection{Poisson Regression Models with Robust Variance to Assess} the Association between SUA Tertiles and Metabolic Syndrome. All regression models are presented in Table 1. In the crude Poisson regression model to calculate the association between SUA tertiles and MetS in the overall population, compared with the low tertile group, the prevalence of MetS in the intermediate and high tertile was higher $(\mathrm{PR}=1.64,95 \% \mathrm{CI}: 1.10-2.46$; $\mathrm{PR}=1.76,95 \% \mathrm{CI}$ : $1.19-2.62$, respectively). Both were statistically significant. In the second model, the association maintained the direction and statistical significance $(\mathrm{PR}=1.60,95 \% \mathrm{CI}$ : $1.07-2.39$;
$\mathrm{PR}=2.04,95 \% \mathrm{CI}: 1.33-3.12$, respectively). In the third model, there was no statistical difference between the intermediate and high tertile compared to the low tertile $(\mathrm{PR}=1.34,95 \%$ CI: 0.92-1.95; PR $=1.40$, 95\% CI: 0.96-2.05, respectively).

When stratified by sex in the second model, men had a higher prevalence of MetS for the intermediate tertile compared to the low tertile ( $\mathrm{PR}=2.36,95 \%$ CI: $1.12-4.95$ ), which was statistically significant. In the third model, the association maintained the pattern $(\mathrm{PR}=1.79,95 \% \mathrm{CI}$ : 0.98-3.23), although there was no statistical difference. Women in the second model had a higher prevalence of MetS for the high tertile compared to the low tertile (PR $=2.43,95 \%$ CI: $1.50-3.93)$, which was statistically significant. In the third model, the association maintained the pattern and statistical significance $(\mathrm{PR}=1.71,95 \% \mathrm{CI}$ : $1.07-2.74)$.

The fully adjusted PRs for each $1 \mathrm{mg} / \mathrm{dl}$ increment in SUA concentration for MetS were 1.22 (95\% CI: 1.04-1.44) in the overall population and 1.24 (95\% CI: 1.01-1.52) in women. In men, no association was found.

3.4. Association between SUA Tertiles and MetS Components and Insulin Resistance Markers. In the adjusted Poisson regression analysis shown in Table 2, the highest tertile of SUA was significantly associated with hypertriglyceridemia (PR: 2.02, 95\% CI: 1.13-3.62) in women, while in men, the middle tertile (PR: 2.27, 95\% CI: 1.22-4.25) and the highest tertile of SUA (PR: 1.94, 95\% CI: 1.01-3.73) presented a significant association to hypertriglyceridemia. In the same way, insulin resistance markers, TG/HDL-C and TyG index, showed elevated levels in the middle tertile of SUA in men. However, in women, only the highest tertile of SUA was associated to elevated TyG index (PR: 1.90, 95\% CI: $1.30-2.76)$.

\section{Discussion}

In the present study, we evaluated the association of SUA to MetS and insulin resistance in health personnel from Peru. Our results showed that health personnel with MetS presented important alterations in anthropometric variables and laboratory tests. Furthermore, hypertriglyceridemia and IR were associated to SUA in both sexes after adjusted analysis. However, only women presented a significant association of SUA to MetS.

SUA is an antioxidant metabolite that maintains the stability of the vascular endothelium [7]. High SUA levels produce a pro-oxidant environment, endothelial dysfunction, and mitochondrial damage. Additionally, the increase of reactive oxygen species (ROS) and inflammatory proteins (interleukin-1, interleukin-6, and TNF- $\alpha$ ) are involved in the development of IR and MetS [9]. Previous studies have shown that elevated SUA levels predispose to IR and MetS $[23,24]$. Some studies infer that high SUA levels may be both a risk factor and an outcome of some metabolic disorders $[25,26]$. 


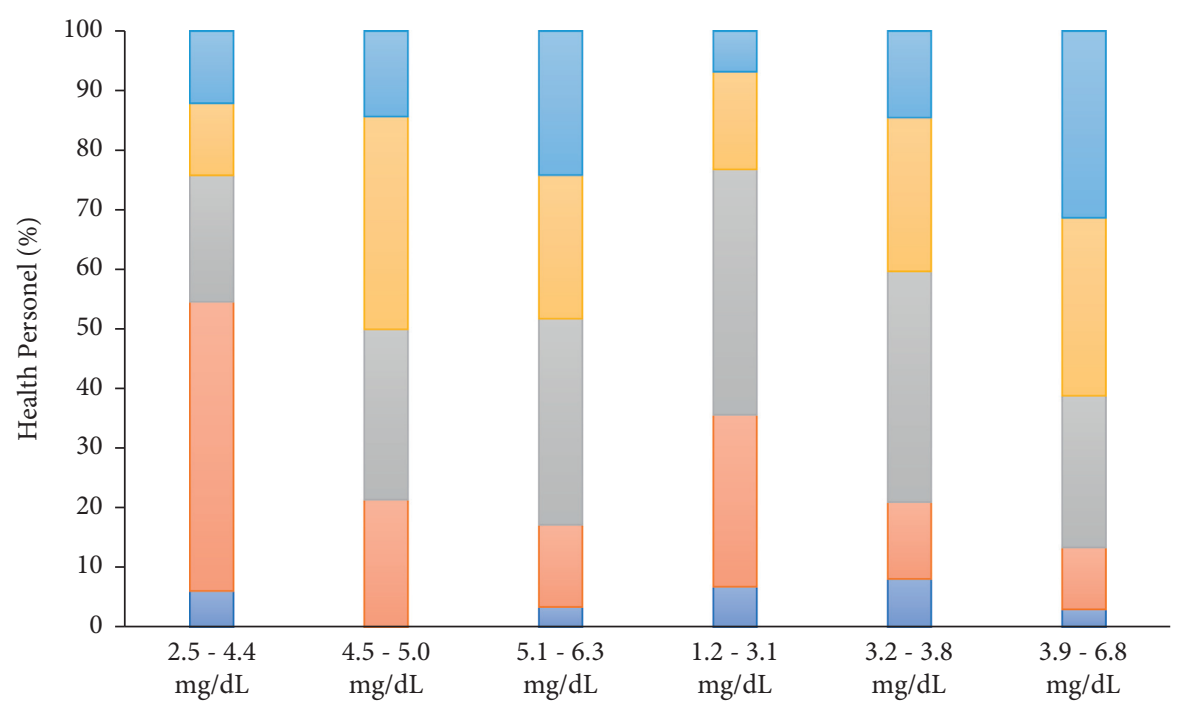

Men Uric acid

Women Uric acid

Metabolic syndrome components

\begin{tabular}{|c|}
\hline$\square 4$ or 5 \\
\hline$\square 3$ \\
\hline
\end{tabular}

FIGURE 1: Components of metabolic syndrome frequency according to tertiles of uric acid. T1 (2.5-4.4 mg/dL), T2 (4.5-5.0 mg/dL), and T3 $(5.1-6.3 \mathrm{mg} / \mathrm{dL})$ in men; T1 $(1.2-3.1 \mathrm{mg} / \mathrm{dL})$, T2 $(3.2-3.8 \mathrm{mg} / \mathrm{dL})$, and T3 $(3.9-6.8 \mathrm{mg} / \mathrm{dL})$ in women.

TAble 1: Prevalence ratio for MetS according to SUA tertiles by overall population and sex.

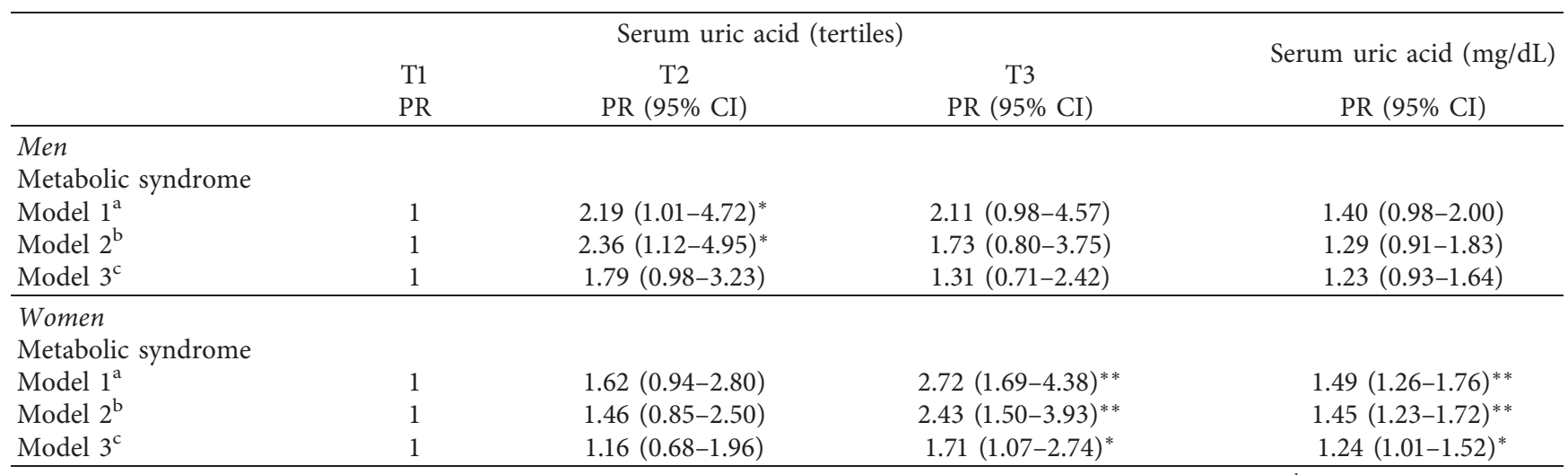

T1: low tertile; T2: middle tertile; T3: high tertile; CI: confidence interval; PR: prevalence ratio; ${ }^{*} p<0.05 ;{ }^{* *} p<0.01$; $^{\text {anonadjusted; }}{ }^{\mathrm{b}}$ adjusted for age; ${ }^{\mathrm{c}}$ adjusted for age, BMI, diabetes family history, physical activity, feeding habits, alcohol consumption, and smoking. Prevalence ratios and confidence intervals were calculated with Poisson regression with robust variance.

TABLE 2: Association between SUA tertiles and MetS components and insulin resistance.

\begin{tabular}{lcccccc}
\hline \multirow{2}{*}{ Metabolic syndrome components } & \multicolumn{2}{c}{ Men } & \multicolumn{2}{c}{ Women $^{2}$} \\
& T1 & T2, PR $(95 \% \text { CI })^{\mathrm{a}}$ & T3, PR (95\% CI) & T1 & T2, PR (95\% CI) & T3, PR (95\% CI) \\
\hline Central obesity & 1 & $1.34(0.64-2.82)$ & $1.13(0.52-2.43)$ & 1 & $1.11(0.64-2.82)$ & $1.23(0.79-1.94)$ \\
High blood pressure & 1 & $0.94(0.27-3.24)$ & $1.00(0.33-3.05)$ & 1 & $0.67(0.17-2.63)$ & $1.53(0.50-4.71)$ \\
Hypertriglyceridemia & 1 & $2.27(1.22-4.25)^{*}$ & $1.94(1.01-3.73)^{*}$ & 1 & $1.32(0.78-2.12)$ & $2.02(1.13-3.20)^{*}$ \\
Hyperglycemia & 1 & $1.18(0.66-2.10)$ & $1.29(0.73-2.25)$ & 1 & $1.02(0.68-1.54)$ & $1.02(0.67-1.53)$ \\
Low HDL-C & 1 & $0.36(0.09-1.46)$ & $0.45(0.13-1.60)$ & 1 & $1.18(0.69-2.01)$ & $1.27(0.75-2.14)$ \\
Elevated TG/HDL-C & 1 & $1.82(1.09-3.04)^{*}$ & $1.67(0.88-3.94)$ & 1 & $1.24(0.70-2.22)$ & $1.44(0.83-2.50)$ \\
Elevated TyG index & 1 & $1.88(1.08-3.30)^{*}$ & $1.39(0.75-3.30)$ & 1 & $1.30(0.85-1.99)$ & $1.90(1.30-2.76)^{* *}$ \\
\hline
\end{tabular}

PR: prevalence ratio; 95\% CI: 95\% confidence interval; TG/HDL-C: triglyceride-to-HDL cholesterol ratio; TyG: triglyceride-glucose index. ${ }^{a}$ Adjusted for HbAlc, LDL-C, and alcohol consumption; ${ }^{*} p<0.05 ;{ }^{* *} p<0.01$. 
The association of SUA to MetS was significant in women but not in men in this study. In this sense, some research studies in Asian populations showed differences in the association of SUA to MetS by sex, with odds of MetS higher in women than men $[27,28]$. Moreover, most studies focus on hyperuricemic populations, while in this study, the population was predominantly normouricemic; this fact could explain our results. On the contrary, some studies found that stratification by age changes the association of SUA to MetS. For example, a study in the Taiwanese military service population showed that elevated SUA women $\geq 40$ years old presented higher odds of MetS than women $<40$ years old [29]. Another study in an older Italian population found that women with high levels of SUA showed a $58 \%$ increased risk of MetS, while in men, no association of SUA to MetS was found [30]. Aging and changes in the endocrine system may explain the susceptibility of women in the development of MetS and high levels of SUA [29]. Besides, estrogens show an inverse relationship with SUA levels, while testosterone increases them. This fact was observed in both sexes [22].

Several studies demonstrate the role of obesity and overweight in the development of cardiometabolic diseases [3-5]. Our results show that individuals with MetS present alterations in anthropometric parameters, BMI, body fat, and central obesity. However, the impact of SUA on MetS components was to increase the frequency of hypertriglyceridemia and not obesity. It is consistent with a longitudinal study in China that found that the highest quartile of SUA had $45.6 \%$ of cumulative incidence to hypertriglyceridemia [31]. In this sense, Jing $\mathrm{Wu}$ et al. showed that uric acid-lowering therapy effectively improved serum cholesterol and triglyceride levels up to $80 \mathrm{mg} / \mathrm{dL}$ [32]. This may be explained by early metabolic changes such as activation of mitochondrial NADPH oxidase and inhibition of AMPK and AKT2, which alter lipid metabolism, mainly triglycerides [9]. Despite obesity is an important factor for the development of MetS, it is likely that disorders in lipid metabolism induced by uric acid develop earlier.

SUA is strongly associated with dyslipidemia and other metabolic disorders, having IR as a common factor [33]. We found that the high tertile of SUA is associated with elevated TyG in both sexes. In the same way, a study performed in Korea showed that the TyG index was significantly higher in the hyperuricemia than in the nonhyperuricemia group (8.96 vs. 8.54, $p<0.001$ ) [34]. Furthermore, a study in ST-elevation myocardial infarction (STEMI) patients demonstrated that the highest quartile of TyG had the incidence of major adverse cardiovascular and cerebral events (MACCEs) which was higher [35]. On the contrary, Elizalde-Barrera et al. did not find any correlation between uric acid levels and homeostatic model assessment of $\beta$-cell function (HOMA 1B) $(r=0.102$, $p=0.343$ ) nor with HOMA of insulin resistance (HOMA 1IR) $(r=0.158, p=0.117)$; when stratified by sex, women had a significant correlation with HOMA $1 \mathrm{IR}(0.278, p=0.01)$, but not with HOMA 1B $(0.138, p=0.257)$ [36].

MetS is a group of insulin-related disorders that increases the risk of multiple diseases such as DM, hypertension, cancer, nonalcoholic fatty liver, chronic kidney disease, brain disorders, and susceptibility to infections $[3,9]$. For this reason, MetS risk factors' research is important to establish therapeutic objectives and primary prevention. Reducing the incidence and prevalence of MetS may help to reduce the risk of developing chronic diseases that demand a high cost for the health system [1]. TyG is an obtainable and cost-effective noninsulin-based IR index that is very useful in primary healthcare [6]. The economic development of Peru has allowed for the adoption of some lifestyles similar to those in high-income countries, such as increased sedentarism, consumption of high-calorie foods, and development of metabolic disorders [37].

Assessing lifestyles with valid instruments is important to establish which factors are relevant to decreased incidence of MetS in susceptible populations such as health personnel, who have an environment with higher levels of stress, depression, burnout syndrome, bad sleep quality, and metabolic disorders $[16,17]$. On the contrary, women appear to be more susceptible to some metabolic disorders than men $[28,38]$. More research is necessary to establish stronger risk factors for MetS in this population.

\section{Strengths and Limitations}

To the best of our knowledge, this is the first study that assessed the association between SUA and MetS conducted in health personnel from Peru. The current study provides evidence on the dose-response relationship between SUA and MetS. Other strengths of our study included the adjustment of several potential confounders. However, some limitations should be highlighted. First, it was not possible to assess causality among the variables due to the nature of the cross-sectional design of the study. Second, although several confounding factors were controlled in the present analysis, there were confounding factors that we did not consider, such as glomerular filtration rate, liver enzymes, and antihyperuricemic agents. Third, information collected from the Plan for the Prevention of Communicable and Noncommunicable Diseases of Hospital de Huaycán was used, which could present some errors when filled in; however, a rigorous evaluation of the quality of the data was carried out to reduce the possibility of biased information. Finally, given that the study was conducted in a single hospital, there is potential for selection bias. Therefore, the results should not be generalized to the population.

\section{Conclusions}

We found evidence that SUA is positively associated with the prevalence of MetS in a population of health personnel at a public hospital from Peru, being the association strongest in women. Additionally, the increased concentration of SUA is an independent factor for hypertriglyceridemia and elevated insulin resistance markers with differences by sex. Longitudinal studies are needed to confirm these results and to determine significant risk factors for MetS considering the cultural environment of each population. More research in lifestyles with valid instruments and additional biomarkers' 
control could decrease the prevalence of MetS and other chronic noncommunicable diseases.

\section{Data Availability}

The datasets used and analyzed for this study are available from the corresponding author upon reasonable request.

\section{Disclosure}

The funder had no role in this work and decided to submit it for publication. A preliminary analysis of this population was presented and accepted in the 18th Annual World Congress Insulin Resistance, Diabetes, and Cardiovascular Disease in the abstracts meeting category.

\section{Conflicts of Interest}

The authors declare that they have no conflicts of interest.

\section{Acknowledgments}

This study was self-funded. ANS was supported by a Fogarty International Center Training Fellowship (TW011502).

\section{Supplementary Materials}

Table S1: title of data: data excluded by implausible or missing values. Description of data: data excluded from the Plan for the Prevention and Surveillance of Communicable and Noncommunicable Diseases by implausible or missing values. Table S2: title of data: general characteristics of the healthcare personnel. Description of data: general characteristics of the healthcare personnel by sex and MetS. (Supplementary Materials)

\section{References}

[1] M. G. Saklayen, "The global epidemic of the metabolic syndrome," Current Hypertension Reports, vol. 20, no. 2, p. 12, 2018.

[2] P. L. Huang, "A comprehensive definition for metabolic syndrome," Disease Models \& Mechanisms, vol. 2, no. 5-6, pp. 231-237, 2009.

[3] S. Furukawa, T. Fujita, M. Shimabukuro et al., "Increased oxidative stress in obesity and its impact on metabolic syndrome," Journal of Clinical Investigation, vol. 114, pp. 17521761, 2017.

[4] C. J. Andersen, K. E. Murphy, and M. L. Fernandez, "Impact of obesity and metabolic syndrome on immunity," Advances in Nutrition, vol. 7, no. 1, pp. 66-75, 2016.

[5] E. Adnan, I. A. Rahman, and H. P. Faridin, "Relationship between insulin resistance, metabolic syndrome components and serum uric acid," Diabetes \& Metabolic Syndrome: Clinical Research \& Reviews, vol. 13, no. 3, pp. 2158-2162, 2019.

[6] N. Babic, A. Valjevac, A. Zaciragic, N. Avdagic, S. Zukic, and S. Hasic, "The triglyceride/HDL ratio and triglyceride glucose index as predictors of glycemic control in patients with diabetes mellitus type 2," Medical Archives, vol. 73, no. 3, pp. 163-168, 2019.
[7] G. Glantzounis, E. Tsimoyiannis, A. Kappas, and D. Galaris, "Uric acid and oxidative stress," Current Pharmaceutical Design, vol. 11, no. 32, pp. 4145-4151, 2005.

[8] J. A. Schmidt, F. L. Crowe, P. N. Appleby, T. J. Key, and R. C. Travis, "Serum uric acid concentrations in meat eaters, fish eaters, vegetarians and vegans: a cross-sectional analysis in the EPIC-oxford cohort," PLoS One, vol. 8, no. 2, Article ID e56339, 2013.

[9] C. King, M. A. Lanaspa, T. Jensen, D. R. Tolan, L. G. SánchezLozada, and R. J. Johnson, "Uric acid as a cause of the metabolic syndrome," Contributions to Nephrology, vol. 192, pp. 88-102, 2018.

[10] C. Caliceti, D. Calabria, A. Roda, and A. Cicero, "Fructose intake, serum uric acid, and cardiometabolic disorders: a critical review," Nutrients, vol. 9, no. 4, p. 395, 2017.

[11] A. F. Rubio-Guerra, H. Morales-López, A. K. GarroAlmendaro et al., "Circulating levels of uric acid and risk for metabolic syndrome," Current Diabetes Reviews, vol. 13, pp. 87-90, 2017.

[12] G. De Pergola, F. Cortese, G. Termine et al., "Uric acid, metabolic syndrome and atherosclerosis: the chicken or the egg, which comes first?" Endocrine, Metabolic \& Immune Disorders-Drug Targets, vol. 18, no. 3, pp. 251-259, 2018.

[13] F. Márquez-Sandoval, G. Macedo-Ojeda, D. ViramontesHörner, J. D. Fernández Ballart, J. Salas Salvadó, and B. Vizmanos, "The prevalence of metabolic syndrome in Latin America: a systematic review," Public Health Nutrition, vol. 14, pp. 1702-1713, 2011.

[14] A. Ruiz-Linares, K. Adhikari, V. Acuña-Alonzo et al., “Admixture in Latin America: geographic structure, phenotypic diversity and self-perception of ancestry based on 7,342 individuals," PLoS Genetics, vol. 10, no. 9, Article ID e1004572, 2014.

[15] M. A. Moreira, A. Vafaei, S. M. A. da Câmara et al., "Metabolic syndrome (MetS) and associated factors in middle-aged women: a cross-sectional study in Northeast Brazil," Women \& Health, vol. 60, no. 6, pp. 601-617, 2020.

[16] B. Vizmanos, A. Betancourt-Nuñez, F. Márquez-Sandoval et al., "Metabolic syndrome among young health professionals in the multicenter Latin America metabolic syndrome study," Metabolic Syndrome and Related Disorders, vol. 18, no. 2, pp. 86-95, 2020.

[17] E. Niazi, M. Saraei, O. Aminian, and N. Izadi, "Frequency of metabolic syndrome and its associated factors in health care workers," Diabetes \& Metabolic Syndrome: Clinical Research \& Reviews, vol. 13, no. 1, pp. 338-342, 2019.

[18] M. V. López, Y. B. Sueldo, C. Gutiérrez, and Y. Angulo-Bazán, "Análisis de la confiabilidad del test Fantástico para medir Estilos de vida saludables en trabajadores evaluados por el Programa "Reforma de Vida"del Seguro Social de Salud (Essalud)," Rev Peru Med Integrativa, vol. 1, p. 17, 2016.

[19] L. Sánchez-Íñigo, D. Navarro-González, A. FernándezMontero, J. Pastrana-Delgado, and J. A. Martínez, "The TyG index may predict the development of cardiovascular events," European Journal of Clinical Investigation, vol. 46, pp. 189197, 2016.

[20] B. Pantoja-Torres, C. J. Toro-Huamanchumo, D. UrrunagaPastor et al., "High triglycerides to HDL-cholesterol ratio is associated with insulin resistance in normal-weight healthy adults," Diabetes \& Metabolic Syndrome: Clinical Research \& Reviews, vol. 13, no. 1, pp. 382-388, 2019.

[21] C. J. Toro-Huamanchumo, D. Urrunaga-Pastor, M. Guarnizo-Poma et al., "Triglycerides and glucose index as an insulin resistance marker in a sample of healthy adults," 
Diabetes \& Metabolic Syndrome: Clinical Research \& Reviews, vol. 13, no. 1, pp. 272-277, 2019.

[22] D. Adamopoulos, C. Vlassopoulos, B. Seitanides, P. Contoyiannis, and P. Vassilopoulos, "The relationship of sex steroids to uric acid levels in plasma and urine," European Journal of Endocrinology, vol. 85, no. 1, pp. 198-208, 1977.

[23] K. Nagahama, T. Inoue, K. Kohagura, A. Ishihara, K. Kinjo, and Y. Ohya, "Hyperuricemia predicts future metabolic syndrome: a 4-year follow-up study of a large screened cohort in Okinawa, Japan," Hypertension Research, vol. 37, no. 3, pp. 232-238, 2014.

[24] M. Liu, Y. He, B. Jiang et al., "Association between serum uric acid level and metabolic syndrome and its sex difference in a Chinese community elderly population," International Journal of Endocrinology, vol. 2014, Article ID 754678, 11 pages, 2014.

[25] J. Dawson and A. Wyss, "Chicken or the egg? Hyperuricemia, insulin resistance, and hypertension," Hypertension, vol. 70, no. 4, pp. 698-699, 2017.

[26] X.-Y. Liu, Q.-Y. Wu, Z.-H. Chen et al., "Elevated triglyceride to high-density lipoprotein cholesterol (TG/HDL-C) ratio increased risk of hyperuricemia: a 4-year cohort study in China," Endocrine, vol. 68, no. 1, pp. 71-80, 2020.

[27] X. Sui, T. S. Church, R. A. Meriwether, F. Lobelo, and S. N. Blair, "Uric acid and the development of metabolic syndrome in women and men," Metabolism, vol. 57, no. 6, pp. 845-852, 2008.

[28] I. Y. Kim, K. D. Han, D. H. Kim et al., "Women with metabolic syndrome and general obesity are at a higher risk for significant hyperuricemia compared to men," Journal of Clinical Medicine, vol. 8, 2019.

[29] Y.-Y. Chen, T.-W. Kao, H.-F. Yang et al., “The association of uric acid with the risk of metabolic syndrome, arterial hypertension or diabetes in young subjects- an observational study," Clinica Chimica Acta, vol. 478, pp. 68-73, 2018.

[30] A. Zurlo, N. Veronese, V. Giantin et al., "High serum uric acid levels increase the risk of metabolic syndrome in elderly women: the PRO.V.A study," Nutrition, Metabolism and Cardiovascular Diseases, vol. 26, no. 1, pp. 27-35, 2016.

[31] Y.-1. Hou, X.-1. Yang, C.-x. Wang, L.-x. Zhi, M.-j. Yang, and C.-g. You, "Hypertriglyceridemia and hyperuricemia: a retrospective study of urban residents," Lipids in Health and Disease, vol. 18, no. 1, p. 81, 2019.

[32] J. Wu, Y. P. Zhang, Y. Qu, L. G. Jie, J. X. Deng, and Q. H. Yu, "Efficacy of uric acid-lowering therapy on hypercholesterolemia and hypertriglyceridemia in gouty patients," International Journal of Rheumatic Diseases, vol. 22, pp. 1445-1451, 2019.

[33] L. Mu, J. Pan, L. Yang, Q. Chen, Y. Chen, and Y. Teng, "Association between the prevalence of hyperuricemia and reproductive hormones in polycystic ovary syndrome," Reproductive Biology and Endocrinology, vol. 16, no. 1, 2018.

[34] J.-S. Byun, J.-N. Kim, Y.-S. Song, Y.-K. Roh, and M.-K. Choi, "The relationship between hyperuricemia and triglyceride glucose index: based on 2016 Korean national health and nutrition examination survey," Korean Journal of Family Practice, vol. 9, no. 3, pp. 266-271, 2019.

[35] E. Luo, D. Wang, G. Yan et al., "High triglyceride-glucose index is associated with poor prognosis in patients with acute ST-elevation myocardial infarction after percutaneous coronary intervention," Cardiovascular Diabetology, vol. 18, no. 1, p. 150, 2019.

[36] C. I. Elizalde-Barrera, T. Estrada-García, J. J. Lozano-Nuevo, A. K. Garro-Almendaro, C. López-Saucedo, and A. F. Rubio-
Guerra, "Serum uric acid levels are associated with homeostasis model assessment in obese nondiabetic patients: HOMA and uric acid," Therapeutic Advances in Endocrinology and Metabolism, vol. 8, no. 10, pp. 141-146, 2017.

[37] J. Medina-Lezama, O. L. Morey-Vargas, H. Zea-Díaz et al., "Prevalence of lifestyle-related cardiovascular risk factors in Peru: the PREVENCION study," Revista Panamericana de Salud Pública, vol. 24, no. 3, pp. 169-179, 2008.

[38] H. Beltrán-Sánchez, M. O. Harhay, M. M. Harhay, and S. McElligott, "Prevalence and trends of metabolic syndrome in the adult U.S. Population, 1999-2010," Journal of the American College of Cardiology, vol. 62, no. 8, pp. 697-703, 2013. 